\title{
Rapid Initiation of Antiretroviral Therapy Following Diagnosis of Human Immunodeficiency Virus Among Patients with Commercial Insurance Coverage
}

\author{
Carmela Benson, MS, MSHP; Bruno Emond, MSc; Patrick Lefebvre, MA; \\ Marie-Hélène Lafeuille, MA; Aurélie Côté-Sergent, MSc; Neeta Tandon, MA; \\ Wing Chow, PharmD, MPH; and Keith Dunn, PharmD, BCPS, AAHIVE
}

\begin{abstract}
BACKGROUND: New guidelines for the treatment of human immunodeficiency virus (HIV) advocate for rapid initiation of antiretroviral therapy (ART) $\leq 7$ days after HIV diagnosis with agents that have a high genetic barrier to resistance, good tolerability, and convenient dosing.
\end{abstract}

OBJECTIVE: To describe characteristics, time to ART initiation, and health care costs in commercially insured patients living with HIV in the United States who are treated $\leq 60$ days after HIV diagnosis.

METHODS: IBM MarketScan Research Databases (January 1, 2012December 31, 2017) were used to identify ART-naive adults with HIV-1, $\geq 6$ months of continuous eligibility before first HIV diagnosis, and ART initiation $\leq 60$ days of first diagnosis. ART regimen had to include a protease inhibitor (PI), an integrase strand transfer inhibitor (INSTI), or a non-nucleoside reverse transcriptase inhibitor (NNRTI) with $\geq 2$ nucleoside reverse transcriptase inhibitors. Cohorts were formed based on time to ART initiation after diagnosis: $\leq 7$ days or 8-60 days. Health care costs were evaluated at $6,12,24$, and 36 months after diagnosis among patients with $\geq 36$ months of continuous eligibility.

RESULTS: Among 9,351 patients, median time to treatment was 31.0 days. Patients initiating ART $>60$ days after HIV diagnosis were excluded $(\mathrm{N}=2,608$ [27.9\%]), while 6,743 (72.1\%) initiated ART $\leq 60$ days after diagnosis and were analyzed; $18.3 \%$ and $81.7 \%$ were classified in the $\leq 7$ days and 8-60 days cohorts, respectively. For all analyzed patients, mean age was $38.0(S D=12.0)$ years and $13.2 \%$ were female; $12.7 \%$, $56.2 \%$, and $31.1 \%$ initiated a PI, INSTI, or NNRTI-based regimen, respectively. Elvitegravir (32.9\%), efavirenz (20.9\%), dolutegravir (18.5\%), and darunavir $(8.5 \%)$ were the most commonly used antiretrovirals; most patients (74.3\%) were initiated on single-tablet regimens. PI-based regimens were more common in the $\leq 7$ days cohort $(\mathrm{PI}=18.1 \%$; darunavir $=11.4 \%)$ than in the $8-60$ days cohort $(\mathrm{PI}=11.5 \%$; darunavir $=7.8 \%$ ). INSTI-based regimens were more common in the 8-60 days cohort (INSTI $=57.7 \%$; elvitegravir $=33.8 \%$ ) than in the $\leq 7$ days cohort (INSTI = 49.2\%; elvitegravir $=29.1 \%$ ). NNRTI-based regimens were as common in the $\leq 7$ days $(32.7 \%)$ and $8-60$ days $(30.7 \%)$ cohorts. Mean total accumulated costs were lower among patients in the $\leq 7$ days cohort than in the 8-60 days cohort at all time points analyzed after diagnosis (e.g., 36 months: $\leq 7$ days $=\$ 109,456 ; 8-60$ days $=\$ 116,870)$. Total per-patient permonth costs decreased over time in the $\leq 7$ days (i.e., 6 months $=\$ 4,359$; 36 months $=\$ 3,040$ ) and 8-60 days cohort ( 6 months $=\$ 4,727$;

36 months $=\$ 3,246)$.

CONCLUSIONS: Although $72.1 \%$ of patients initiated ART $\leq 60$ days after HIV diagnosis, only $18.3 \%$ initiated ART $\leq 7$ days. Many patients initiating ART $\leq 7$ days used suboptimal agents with low rather than high genetic barriers to resistance (i.e., efavirenz and elvitegravir) or agents (dolutegravir) coformulated with other antiretrovirals that require testing to prevent hypersensitivity reactions. Patients in the $\leq 7$ days cohort showed lower total health care costs relative to those in the 8-60 days cohort, highlighting the potential long-term benefits of rapid ART initiation.

J Manag Care Spec Pharm. 2020;26(2):129-41

Copyright $\odot 2020$, Academy of Managed Care Pharmacy. All rights reserved.

\section{What is already known about this subject}

-When used optimally, antiretroviral therapy (ART) effectively reduces the risk of transmission and improves clinical outcomes and quality of life in people living with human immunodeficiency virus (HIV).

There is now compelling evidence supporting that ART should be initiated as soon as possible in individuals with HIV, hence, the recommendation of the World Health Organization (WHO) to initiate ART at any given CD4 count and $\leq 7$ days after HIV diagnosis.

There is a need to complement previous findings from a Medicaid-insured population and to assess the real-world characteristics, time to ART initiation, and health care costs of commercially insured patients with HIV stratified by timeliness of ART initiation.

\section{What this study adds}

Less than 20\% of ART-treated commercially insured patients with HIV initiated ART $\leq 7$ days after HIV diagnosis as recommended by current WHO guidelines.

Many patients who initiated ART $\leq 7$ days used suboptimal agents with low rather than high genetic barriers to resistance (i.e., efavirenz and elvitegravir).

Regardless of the length of follow-up considered, total accumulated and per-patient per-month costs were consistently lower among patients who initiated ART $\leq 7$ days following HIV diagnosis relative to those initiated from 8-60 days, suggesting that rapid initiation of ART may be associated with cost savings for payers. 
$\mathrm{H}$ uman immunodeficiency virus (HIV) is a chronic and incurable infectious disease characterized by a decline in the number of CD4-positive T cells, which underlies the immunosuppression observed in affected individuals. ${ }^{1}$ In 2015, 1.2 million individuals were living with HIV in the United States. ${ }^{2}$ When used optimally, antiretroviral therapy (ART) effectively reduces the risk of transmission and improves clinical outcomes and quality of life in people living with HIV (PLWH). ${ }^{3-8}$ However, there are societal and administrative barriers preventing rapid access to diagnosis and treatment, thereby considerably hindering efforts to reduce the burden of HIV. The objective set by the United Nations-also known as the 90-90-90 targets-is to have 90\% of the HIV-infected population diagnosed, $90 \%$ of those diagnosed treated with ART, and $90 \%$ of those treated with ART to have viral suppression by 2020. ${ }^{9}$

For several years, the optimal timing of ART initiation has been a matter of debate. The first evidence on the benefits of early ART initiation came from cohort studies, which showed that CD4-positive T cell counts at the start of ART strongly predicted the risk of death or progression to acquired immunodeficiency syndrome (AIDS). ${ }^{10}$ Randomized clinical trials, including the SMART, TEMPRANO, INSIGHT, and HPTN 052 trials, have convincingly established the benefits of early ART initiation. ${ }^{4,11-14}$ Subsequent studies demonstrated that strategies to initiate ART more rapidly or on the same day as HIV testing positively affect clinical outcomes and retention in care. ${ }^{15-19}$ Based on this evidence, the 2017 World Health Organization (WHO) guidelines recommend initiating ART at any given CD4 count and $\leq 7$ days after HIV diagnosis (i.e., rapid initiation). ${ }^{20}$ In the United States, the Centers for Disease Control and Prevention estimates that $86 \%$ of PLWH are diagnosed, $65 \%$ have received care, $49 \%$ are retained in care (defined as having $\geq 2$ viral load or CD4-positive tests at least 3 months apart), and $51 \%$ have achieved viral suppression. ${ }^{21}$

Although treating all PLWH earlier may entail additional pharmacy costs, these must be balanced with the long-term clinical benefits of this policy, which may allow patients to live longer and develop fewer immune-related complications, thus leading to potentially lower medical costs. However, studies conducted to date and related to rapid initiation of ART were not real-world studies or used non-U.S.-specific data. ${ }^{17-19}$ Recently, a retrospective longitudinal analysis found that only $20.4 \%$ of ART-treated Medicaid-insured patients started ART $\leq 14$ days after HIV diagnosis and that these patients incurred lower costs compared with those initiating ART later. ${ }^{31}$ However, the timeliness of ART initiation (which may be different than in Medicaid-insured patients) and the economic implications of rapid ART initiation have not been assessed among commercially insured patients, who represent a greater proportion of the HIV population and have different characteristics. ${ }^{32}$ Therefore, there is a need to complement the findings from the Medicaid-insured population and to assess the real-world costs incurred by commercially insured PLWH stratified by timeliness of ART initiation. This study aims to describe characteristics, time to ART initiation, and health care costs of commercially insured PLWH treated $\leq 7$ days ( $\leq 7$ days cohort) and 8-60 days (8-60 days cohort) following their initial HIV diagnosis.

\section{Methods}

\section{Data Sources}

The IBM MarketScan Research Databases were used (January 1, 2012-December 31, 2017). These longitudinal databases comprise the Commercial Claims and Encounters database and the Medicare Supplemental and Coordination of Benefits database, which include the combined claims from approximately 100 payers (i.e., commercial insurance companies, Blue Cross and Blue Shield plans, and third-party administrators). The data come from a selection of large employers, health plans, and government and public organizations that represent nearly 240 million covered lives since 1995, including employees and their dependents, self-insured employers, and Medicare-eligible retirees with employer-provided Medicare supplemental plans. The databases cover all U.S. census regions, with concentration in the South and North Central (Midwest) regions. All data collected were deidentified in compliance with the patient confidentiality requirements of the Health Insurance Portability and Accountability Act (HIPAA). As this was a retrospective analysis of existing claims data and no patient-identifiable information was included in the claims dataset, institutional review board approval was not required. ${ }^{33}$

\section{Study Design}

A retrospective longitudinal study design was used to analyze patients from January 1, 2012-December 31, 2017. The index date was defined as the date of the first HIV-1 diagnosis (International Classification of Diseases, Ninth Revision, Clinical Modification [ICD-9-CM]: 042, V08, and 795.71; International Classification of Diseases, Tenth Revision, Clinical Modification [ICD-10-CM]: B20, R75, and Z21) during the study period (Appendix, available in online article). The baseline period was defined as the first 6 months before the index date. The follow-up period spanned from the index date up until the end of data availability, which included plan disenrollment, loss of follow-up, or data cut-off (Appendix). Clinical characteristics (i.e., opportunistic infections and mental health-related comorbidities) were assessed during the 6-month pre-ART period. Among patients with $\geq 36$ months of follow-up post-index, health care costs were assessed during fixed time periods of 6 , 12,24 , and 36 months. 


\section{Patient Selection}

Inclusion Criteria. Included patients were required to have received an antiretroviral agent as part of an ART regimen $\leq 60$ days after HIV diagnosis, be $\geq 18$ years old, have $\geq 6$ months of continuous insurance eligibility pre-index, and have no claims for an antiretroviral agent other than pre-exposure prophylaxis (defined as having a claim for the fixed-dose combination emtricitabine/tenofovir disoproxil fumarate) at any time preHIV diagnosis. An ART regimen was defined as having a claim for a protease inhibitor (PI), an integrase strand transfer inhibitor (INSTI), or a non-nucleoside reverse transcriptase inhibitor (NNRTI), together with $\geq 2$ different nucleoside reverse transcriptase inhibitors, with each claim separated by no more than 14 days. Regimens with or without a pharmacological booster were included. Patients with hepatitis B were not excluded.

Exclusion Criteria. Patients who received a diagnosis of HIV-2 (ICD-9-CM: 079.53; ICD-10-CM: B97.35) at any time and patients who had their first HIV diagnosis recorded during an inpatient stay that lasted $\geq 10$ days were excluded. This latter criterion aimed to take into account that some medical services received during an inpatient stay, such as ART initiation, may not be recorded. Patients initiating ART $>60$ days after HIV diagnosis were excluded from the study after a feasibility analysis revealed that a majority were initiated on ART $\leq 60$ days of diagnosis.

Cohort Definition. Patients meeting all selection criteria were classified into mutually exclusive cohorts based on timeliness of receipt of ART after HIV diagnosis: $\leq 7$ days (i.e., per WHO definition of rapid initiation) and 8-60 days. ${ }^{20}$

\section{Outcome Measures and Statistical Analysis}

Time to ART initiation was evaluated with descriptive statistics of the cumulative proportion of patients initiated on ART. Patient demographics (i.e., age, gender, U.S. region, insurance type) were evaluated at the index date. The type of first ART regimen initiated post-index was also evaluated. Quan-Charslon Comorbidity Index (Quan-CCI), opportunistic infections (i.e., diagnosis for pneumocystis pneumonia, toxoplasma gondii encephalitis, mycobacterium tuberculosis infection, disseminated mycobacterium avium complex disease, histoplasma capsulatum infection, coccidiodomycosis, cryptococcosis, or cytomegalovirus disease), pathology and laboratory procedures, and Diagnostic and Statistical Manual of Mental Disorders, 5th Edition comorbidities for which $\geq 5 \%$ of patients received a diagnosis in $\geq 1$ study cohort were evaluated during the first 6 months before initiation of ART to identify their relationship with time to ART initiation. Total all-cause health care costs during the 6-, 12-, 24-, and 36-month periods post-index were evaluated among patients with $\geq 36$ months of insurance eligibility after HIV diagnosis and included accumulated and per-patient per-month (PPPM) health care costs, each stratified into all-cause pharmacy and all-cause medical costs (i.e., sum of emergency department, inpatient, outpatient, and others services [i.e., durable medical equipment use and dental/vision care] costs).

All study measures were descriptively evaluated. Means and standard deviations (SDs) were used to describe continuous variables, and frequencies and percentages were used for categorical variables. All costs were reported in constant 2017 U.S. dollars using the medical care component of the Consumer Price Index. ${ }^{34}$

\section{Results}

Timeliness of ART Initiation and Types of ART Regimen Initiated Of 41,638 eligible patients with an HIV diagnosis, 9,351 (22.5\%) received an eligible ART regimen any time after diagnosis (Figure 1). Among these patients, median time to ART initiation was 31.0 days; mean age was 37.8 years $(S D=12.0)$, mean Quan-CCI was 0.07 ( $\mathrm{SD}=0.35)$, and $8.6 \%, 7.9 \%$, and $9.3 \%$ of patients had anxiety disorders, depressive disorders, and substance-related and addictive disorders, respectively.

Since most patients initiated ART in the first 2 months after HIV diagnosis, and to focus on patients initiating treatment rapidly, the 2,608 (27.9\%) patients initiating an eligible ART regimen $>60$ days after HIV diagnosis were excluded, while the remaining 6,743 (72.1\%) patients initiating ART $\leq 60$ days after HIV diagnosis were included in the present study. A total of 1,232 (18.3\%) and 5,511 (81.7\%) patients were classified in the $\leq 7$ days and 8-60 days cohorts, respectively (Figure 1).

The cumulative proportions of patients initiated on ART after HIV diagnosis were $18.3 \%, 64.4 \%$, and $100.0 \%$ at 1,4 , and 8 weeks after diagnosis, respectively (Figure 2). In comparison, in a population of Medicaid-insured patients, the cumulative proportions of patients initiated on ART after HIV diagnosis were $12.8 \%, 35.6 \%$, and $56.8 \%$ at 1,4 , and 8 weeks, respectively (Figure 2). ${ }^{35}$

Overall, $12.7 \%, 56.2 \%$, and $31.1 \%$ of patients were initiated on a PI, INSTI, and NNRTI-based regimen, respectively (Table 1). PI-based regimens appeared to be more commonly used among patients initiated $\leq 7$ days compared with those initiated 8-60 after HIV diagnosis (Table 1). Darunavir was the most commonly used agent among PIs, and its use was more common in the $\leq 7$ days cohort relative to the 8-60 days cohort (Table 1). INSTI-based regimens were less common in patients initiating treatment $\leq 7$ days relative to those initiated 8-60 days after diagnosis (Table 1), with the most commonly used agent in this class (i.e., elvitegravir) following the same pattern of use observed in the overall category (Table 1). Of all INSTIs evaluated, dolutegravir-based regimens had the most pronounced difference in use between the $\leq 7$ days cohort and 8-60 days cohort (Table 1). A proportion of $61.4 \%$ of patients treated with dolutegravir had a dolutegravir/abacavir coformulation; $93.5 \%$ of patients who received this coformulation were 
Rapid Initiation of Antiretroviral Therapy Following Diagnosis of Human Immunodeficiency Virus Among Patients with Commercial Insurance Coverage

\section{FIGURE 1 Identification of Study Population}

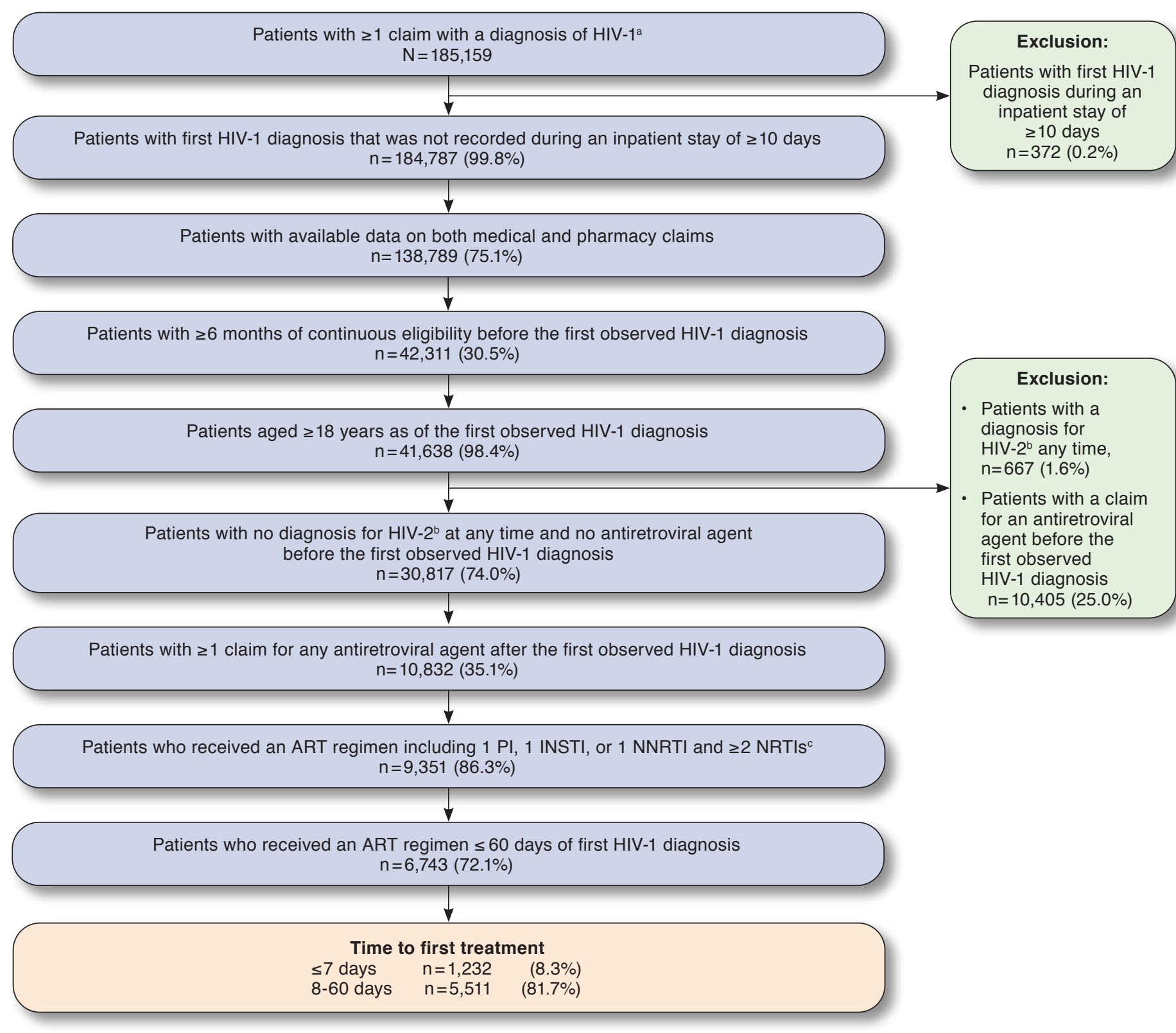

a HIV-1 diagnosis was identified using ICD-9-CM codes 042, 795.71, and V08 and ICD-10-CM codes B20, R75, and Z21.

bHIV-2 diagnosis was identified using ICD-9-CM code 079.53 and ICD-10-CM code B97.35.

cAn ART regimen was defined based on claims filled within 14 days from the index date.

$A R T$ = antiretroviral therapy; HIV = human immunodeficiency virus; ICD-9-CM/ICD-10-CM=International Classification of Disease, Ninth/Tenth Revision, Clinical

Modification; INSTI = integrase strand transfer inhibitor; NNRTI=non-nucleoside reverse transcriptase inhibitor; NRTI=nucleoside reverse transcriptase inhibitor;

PI= protease inhibitor

in the 8-60 days cohort. NNRTI-based regimens appeared to be as commonly used among patients initiating ART $\leq 7$ days as in patients initiating ART 8-60 days after HIV diagnosis (Table 1).

\section{Demographic and Clinical Characteristics}

Among all patients and at the time of the first HIV diagnosis, mean age was 38.0 years, and $13.2 \%$ were female (Table 1). Most patients resided in the South region and most had a 
Cumulative proportion of commercially insured patients initiated on ART since first HIV diagnosis ${ }^{a}$ $(\mathrm{N}=6,743)$

Cumulative proportion of Medicaid-insured patients initiated on ART since first HIV diagnosis ${ }^{b}$ $(\mathrm{N}=627)$

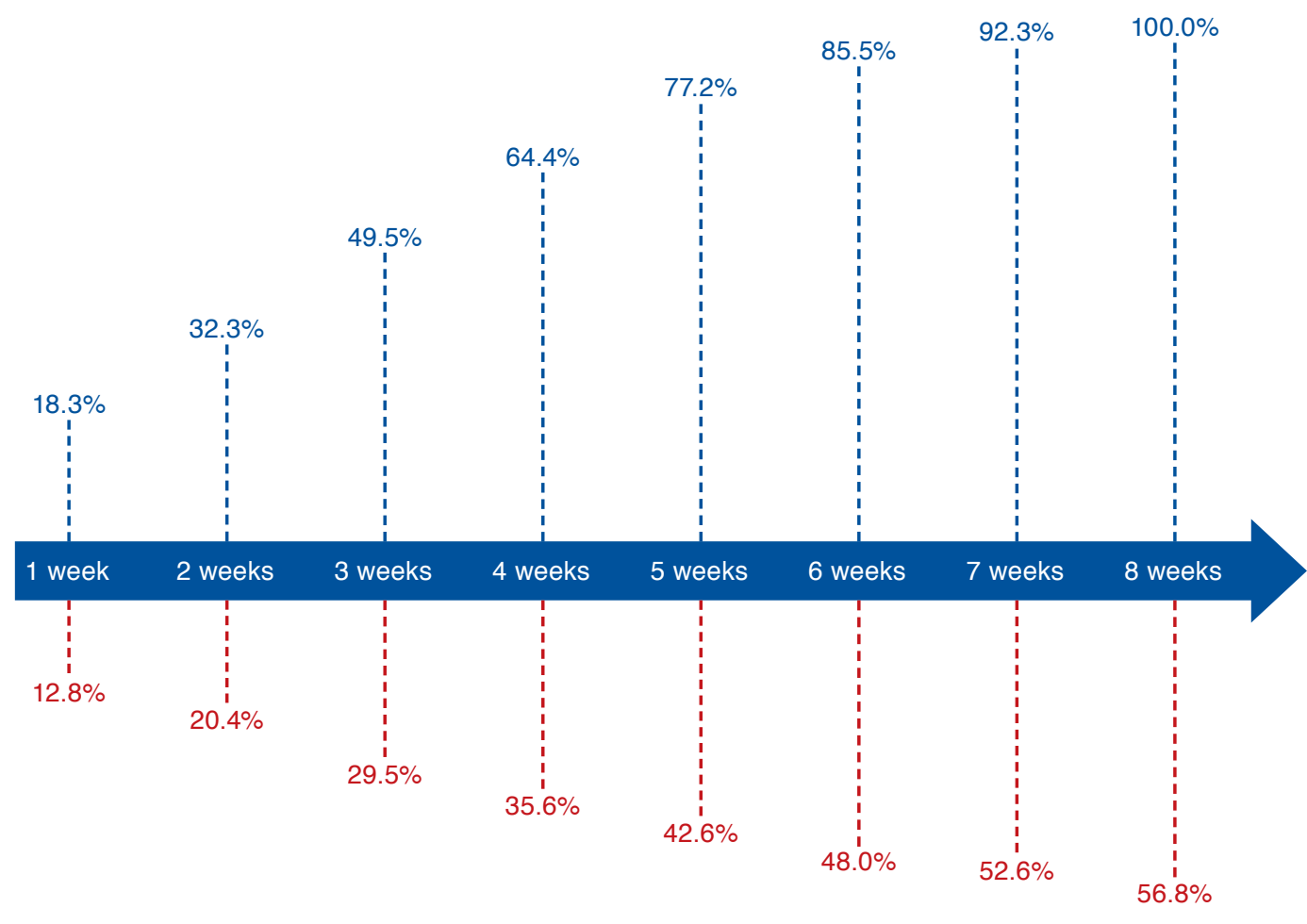

adult patients with HIV who initiated an ART from Truven Health MarketScan Research Databases 21 2012-Q4 2017 were included.

bAdult patients with HIV who initiated an ART from Medicaid databases from 6 states (Iowa [Q1 2012-Q1 2017], Kansas [Q1 2012-Q1 2017], New Jersey [Q1 2012-Q1 2014], Missouri [Q1 2012-Q1 2017], Mississippi [Q1 2012-Q1 2017], and Wisconsin [Q1 2012-Q4 2013]) were included.

$A R T=$ antiretroviral therapy; HIV = human immunodeficiency virus.

preferred provider organization health care plan (Table 1). Demographic characteristics did not substantially differ between the $\leq 7$ days and 8-60 days cohorts; however, this was not the case for clinical characteristics such as the proportion of patients who received a single-tablet regimen (STR) $(\leq 7$ days $=63.9 \% ; 8-60$ days $=76.6 \%)$ and the proportion of patients with an opportunistic infection $(\leq 7$ days $=2.8 \% ; 8-60$ days $=7.7 \%$; Table 1 ).

During the 6-month period before ART initiation, a numerically lower proportion of patients in the $\leq 7$ days cohort had any mental health-related comorbidities compared with the 8-60 days cohort (Table 1). More specifically, anxiety disorders, depressive disorders, substance-related and addictive disorders, and sleep-wake disorders were less frequent among the $\leq 7$ days cohort relative to the 8-60 days cohort (Table 1 ).

\section{Total Accumulated Medical Costs After HIV Diagnosis}

A total of 194 and 1,018 patients from the $\leq 7$ days cohort and 8-60 days cohort had $\geq 36$ months of follow-up after HIV diagnosis, respectively, and were included in the cost analysis.
After 6 months following the HIV diagnosis, mean per-patient accumulated medical costs were $\$ 10,574$ among patients initiating ART $\leq 7$ days and $\$ 13,438$ among those initiating ART 8-60 days after diagnosis (Figure 3). This trend was similar when considering follow-up durations of 12 months, 24 months, and 36 months (Figure 3). Inpatient (36 months: $\leq 7$ days $=41.2 \% ; 8-60$ days $=44.7 \%)$ and outpatient (36 months: $\leq 7$ days $=50.3 \%$; $8-60$ days $=48.6 \%$ ) costs were the 2 main drivers of medical costs in both study cohorts at all time points analyzed.

\section{Total Accumulated Health Care Costs After HIV Diagnosis}

The total all-cause accumulated health care costs of patients who received ART $\leq 7$ days after diagnosis were consistently lower than those of patients who received treatment 8-60 days after diagnosis over follow-up periods of $6,12,24$, and 36 months (Figure 3). The proportion of total costs attributable to medical costs was consistently higher among patients in the 8-60 days cohort relative to patients in the $\leq 7$ days cohort (Figure 3). Pharmacy costs were higher for patients 
Rapid Initiation of Antiretroviral Therapy Following Diagnosis of Human Immunodeficiency Virus Among Patients with Commercial Insurance Coverage

\section{TABLE 1 Demographic and Clinical Characteristics}

Time to treatment (days), mean \pm SD

Age at HIV diagnosis (years)a, mean \pm SD

Female, \%

Region, \%

North East

North Central

South

West

Unknown

Type of commercial health care plana, \%

$\mathrm{PPO}$

Comprehensive

$\mathrm{HMO}$ and POS with capitation

POS and EPO

CDHP and HDHP

Unknown

Patients who initiated a single-tablet regimen, \%

ART regimen received, \%

PI-based regimen

Darunavir-based regimen

NNRTI-based regimen

Efavirenz-based regimen

INSTI-based regimen

Dolutegravir-based regimen

Elvitegravir-based regimen

Patients with $\geq 1$ diagnosis of an opportunistic infectionb,c, \%

Quan CCI (excluding HIV symptoms) $)^{\mathrm{b}}$, mean \pm SD

Patients with mental health-related comorbidities ${ }^{\mathrm{b}}, \%$

Any mental health-related comorbidity

Anxiety disorders

Depressive disorders

Substance-related and addictive disorders

Sleep-wake disorders

Location of first HIV diagnosis ${ }^{\mathrm{a}}$ \%

Emergency room

Inpatient

Pathology and laboratory procedures and services received ${ }^{\mathrm{b}}, \%$

Infectious agent detection by nucleic acid

Complete blood count

Comprehensive metabolic panel

T-cells: absolute CD4 and CD8 count

Syphilis test, non-treponemal antibody

Lipid panel

Hepatitis B surface antibody

Hepatitis $\mathrm{C}$ antibody

Infectious agent antigen detection by immunoassay technique

Urinalysis

a Assessed on the index date.

${ }^{b}$ Assessed during the 6-month period before ART initiation.

cThe opportunistic infections considered were pneumocystis pneumonia, toxoplasma gondii encephalitis, mycobacterium tuberculosis infection, disseminated mycobacterium avium complex disease, histoplasma capsulatum infection, coccidioidomycosis, cryptococcosis, and cytomegalovirus disease.

$A R T=$ antiretroviral therapy; $C C I=$ Charlson Comorbidity Index; $C D H P=$ consumer-directed health plan; $E P O=$ exclusive provider organization; HDHP=high-deductible health plan; HIV= human immunodeficiency virus; HMO=health maintenance organization; INSTI=integrase strand transfer inhibitor; NNRTI =non-nucleoside reverse transcriptase inhibitor; $P I=$ protease inhibitor; $P O S=$ point of service; $P P O=$ preferred provider organization; $S D=$ standard deviation.

\begin{tabular}{|r|r|r|}
\hline 14.1 & 16.2 & 13.6 \\
\hline 13.1 & 12.0 & 13.3 \\
\hline 56.6 & 51.7 & 57.7 \\
\hline 15.2 & 19.2 & 14.4 \\
\hline 1.0 & 0.9 & 1.1 \\
\hline
\end{tabular}

\begin{tabular}{|r|r|r|}
\hline 55.6 & 56.0 & 55.5 \\
\hline 2.1 & 2.8 & 1.9 \\
\hline 12.8 & 16.6 & 11.9 \\
\hline 9.7 & 8.1 & 10.0 \\
\hline 17.7 & 14.0 & 18.5 \\
\hline 2.2 & 2.6 & 2.1 \\
\hline 74.3 & 63.9 & 76.6 \\
\hline
\end{tabular}

12.7

8.5

31.1

20.9

56.2

18.5

32.9

6.8

$0.07 \pm 0.35$

\begin{tabular}{c}
18.1 \\
11.4 \\
32.7 \\
\hline 24.0 \\
\hline 49.2 \\
13.5 \\
\hline 29.1 \\
\hline 2.8 \\
\hline $.07 \pm 0.33$
\end{tabular}

11.5

7.8

30.7

20.2

57.7

19.7

33.8

7.7

$0.07 \pm 0.35$

\begin{tabular}{|r|r}
\hline 30.3 & \\
\hline 8.5 & \\
\hline 7.5 & \\
\hline 8.9 & \\
\hline 5.3 & \\
\hline
\end{tabular}

\begin{tabular}{r|r}
\hline 4.0 & 4.9 \\
\hline 11.3 & 6.3 \\
\hline
\end{tabular}

3.8

\begin{tabular}{|r|r|r|}
\hline 30.3 & 23.9 & 31.7 \\
\hline 8.5 & 5.6 & 9.1 \\
\hline 7.5 & 5.3 & 7.9 \\
\hline 8.9 & 7.1 & 9.3 \\
\hline 5.3 & 4.5 & 5.4 \\
\hline \multicolumn{3}{|c|}{} \\
\hline 4.0 & 4.9 & 3.8 \\
\hline 11.3 & 6.3 & 12.4 \\
\hline 67.1 & 41.1 & 73.0 \\
\hline 59.3 & 43.1 & 62.9 \\
\hline 65.2 & 44.1 & 69.9 \\
\hline 42.5 & 25.0 & 46.5 \\
\hline 56.2 & 35.6 & 60.8 \\
\hline 47.5 & 30.4 & 51.4 \\
\hline 39.2 & 20.3 & 43.5 \\
\hline 42.8 & 26.3 & 46.5 \\
\hline 38.1 & 21.9 & 41.7 \\
\hline 27.2 & 18.0 & 29.2 \\
\hline
\end{tabular}

\begin{tabular}{c|c|c|}
$\begin{array}{c}\text { All Patients } \\
\mathbf{N}=6,743\end{array}$ & $\begin{array}{c}\leq 7 \text { Days } \\
\mathbf{n}=\mathbf{1}, 232\end{array}$ & $\begin{array}{c}8-60 \text { Days } \\
\mathbf{n}=5,511\end{array}$ \\
\hline $23.6 \pm 15.8$ & $2.4 \pm 2.6$ & $28.3 \pm 13.5$ \\
\hline $38.0 \pm 12.0$ & $38.8 \pm 12.2$ & $37.8 \pm 11.9$ \\
\hline 13.2 & 14.0 & 13.0 \\
\hline
\end{tabular}




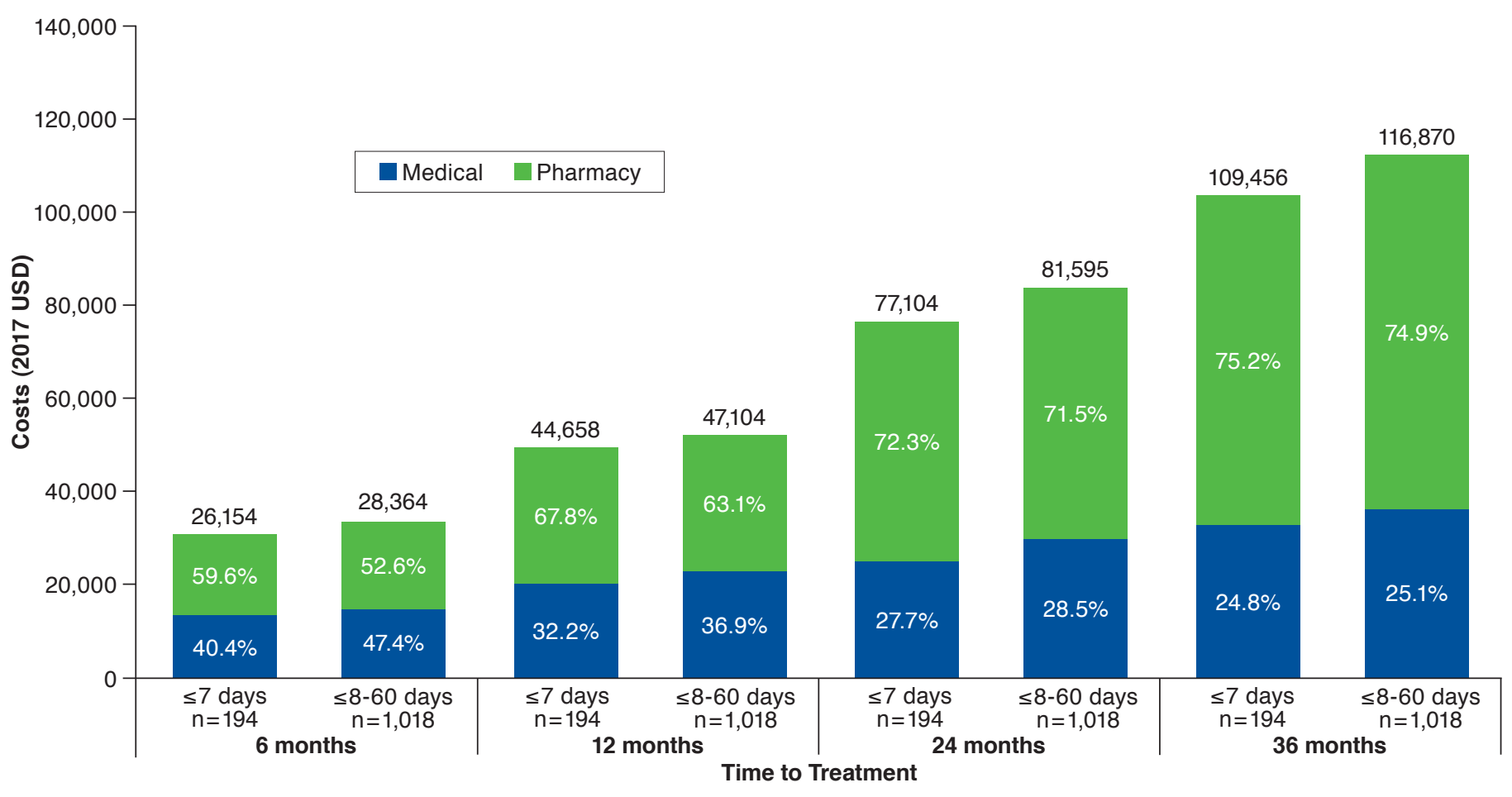

aTotal accumulated health care costs correspond to the sum of pharmacy and medical costs incurred over the 6-, 12-, 24-, or 36-month periods after HIV diagnosis. Medical costs include costs related to outpatient visits, inpatient visits, emergency department visits, and other services (i.e., durable medical equipment use and dental or vision care).

HIV = human immunodeficiency virus; USD = U.S. dollars.

in the $\leq 7$ days cohort compared with those in the 8-60 days cohort after $6(\leq 7$ days $=\$ 15,580 ; 8-60$ days $=\$ 14,925)$ and 12 $(\leq 7$ days $=\$ 30,275 ; 8-60$ days $=\$ 29,719)$ months of followup, but the inverse was observed when considering follow-up periods of 24 ( $\leq 7$ days $=\$ 55,777 ; 8-60$ days $=\$ 58,346)$ and 36 months ( $\leq 7$ days $=\$ 82,303 ; 8-60$ days $=\$ 87,516)$.

\section{Monthly Total Health Care Costs After HIV Diagnosis}

Patients initiated on ART $\leq 7$ and 8-60 days after diagnosis incurred decreasing total PPPM costs after 6, 12, 24, and 36 months of follow-up (Figure 4). In the $\leq 7$ days cohort, PPPM medical costs decreased from $\$ 1,762$ after 6 months to $\$ 754$ after 36 months, and PPPM pharmacy costs decreased from $\$ 2,597$ after 6 months to $\$ 2,286$ after 36 months. In the 8-60 days cohort, medical costs decreased from $\$ 2,240$ after 6 months to $\$ 815$ after 36 months, and pharmacy costs decreased from $\$ 2,488$ after 6 months to \$2,431 after 36 months (Figure 4). The proportion of total costs attributable to medical costs decreased from $40.4 \%$ after 6 months to $24.8 \%$ after 36 months of follow-up in the $\leq 7$ days cohort and from $47.4 \%$ to $25.1 \%$ in the $8-60$ days cohort.

\section{Discussion}

In this retrospective study, patient characteristics, timeliness of ART initiation, and health care costs of ART-treated commercially insured PLWH were evaluated among those initiating ART $\leq 7$ days and 8-60 days after diagnosis. Only 18.3\% of patients were initiated on ART $\leq 7$ days as recommended in the most recent WHO guidelines, suggesting that this recommendation is not optimally implemented among commercially insured patients. ${ }^{20}$

INSTI-based regimens were less commonly used in the $\leq 7$ days cohort compared with the 8-60 days cohort. In contrast, PI-based regimens were used more frequently in the $\leq 7$ days cohort versus the 8-60 days cohort. Mental health-related comorbidities were more frequent in the 8-60 days cohort compared with the $\leq 7$ days cohort. Regardless of the length of follow-up considered, total accumulated and PPPM costs were consistently lower among patients initiated on ART $\leq 7$ days compared with those initiated on ART 8-60 days after HIV diagnosis. In both cohorts, total PPPM health care costs decreased with longer follow-up periods, which was mostly driven by reductions in PPPM medical costs. 


\section{FIGURE 4 Health Care Costs PPPM in the First 6, 12, 24, and 36 Months After HIV Diagnosis}

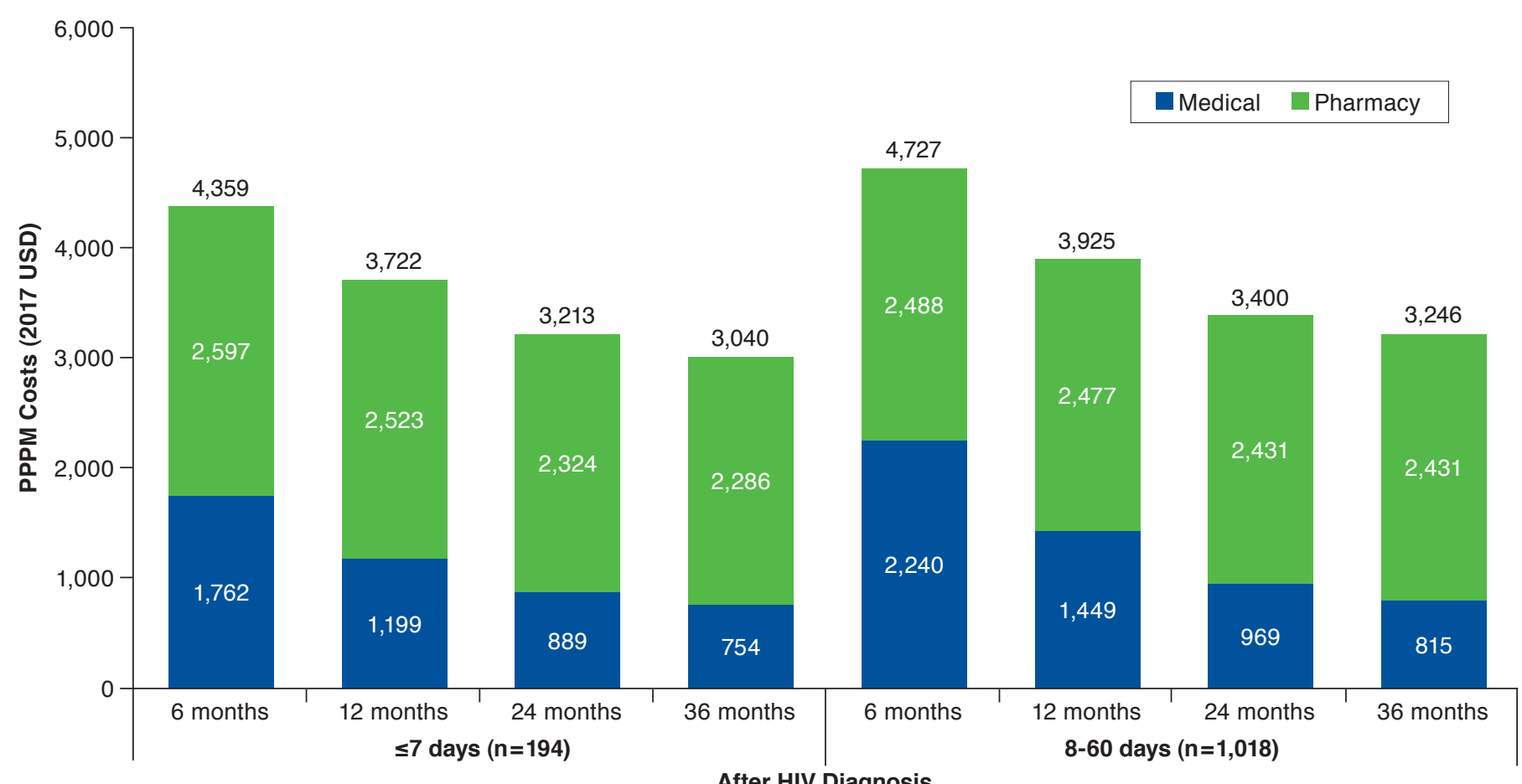

After HIV Diagnosis

HIV=human immunodeficiency virus; PPPM=per-patient per-month; USD=U.S. dollars.

Recommendations for ART initiation have substantially evolved over the past few years. In 2010, the WHO recommended to initiate ART when CD4 counts were below 350 cells/ $\mu \mathrm{L}$, largely based on results from the CIPRAHT-001 and SMART trials. ${ }^{11,36,37}$ This recommendation was subsequently revised to a threshold of 500 CD4-positive cells/ $\mu \mathrm{L}$ in 2013, before the current recommendation of initiating ART at any given CD4 count was formulated in 2015..$^{38,39}$ The latter guidance was based on data from the TEMPRANO (immediate vs. initiation at $\leq 500$ CD4-positive cells/ $\mu \mathrm{L}$ ), INSIGHT (immediate vs. initiation at $\leq 350$ CD4-positive cells/ $\mu \mathrm{L}$ ), and HPTN 052 (immediate vs. initiation at $\leq 250$ CD4-positive cells/uL or AIDS) trials, which largely contributed to establishing this new standard of care in the treatment of HIV.,12-14 Instead of thresholds based on CD4 counts, current WHO guidelines recommend initiating ART $\leq 7$ days after HIV diagnosis, and the Department of Health and Human Services (DHHS) guidelines recommend immediate initiation of ART regardless of CD4 counts. ${ }^{20}$

In spite of the solid evidence backing this recommendation, only $18.3 \%$ of ART-treated patients in the current study were initiated on ART $\leq 7$ days after diagnosis. This proportion is consistent with a recent study of Medicaid-insured patients, where 20.4\% of ART-treated patients were initiated on ART $\leq 14$ days after diagnosis. ${ }^{31}$ However, the latter Medicaid population included $43.2 \%$ of patients initiated on ART $>60$ days after diagnosis, whereas fewer patients had such delays in the current study (i.e., 27.9\%).

While it appears that rapid ART initiation is more common in commercially insured PLWH, these results suggest that the recommendation to initiate ART $\leq 7$ days is not optimally implemented both in Medicaid- and commercially insured patients. This may signal the existence of societal and administrative barriers precluding rapid access to treatment. It should be noted, however, that a majority of patients included in the present study initiated ART before the publication of new guidance recommending rapid ART initiation. Further research is warranted to understand the factors underlying delayed treatment initiation.

In the present study, a relatively high proportion (56.2\%) of patients was initiated on an INSTI-based regimen. With the exception of dolutegravir-based regimens, treatment guidelines from DHHS do not recommend INSTI-based regimens for rapid initiation of ART. ${ }^{40}$ Results from this study show that $35.7 \%$ of patients in the $\leq 7$ days cohort (calculated as 
the difference between the proportion of patients initiating an INSTI-based regimen and the proportion of patients initiating a dolutegravir-based regimen in the $\leq 7$ days cohort) initiated other-than-recommended INSTI-based regimens, thus demonstrating that there remains a possibility to improve regimen selection and HIV care.

Although guidelines recommend dolutegravir-based regimens in rapid initiation, the proportion of patients using this regimen was higher in the 8-60 days cohort compared with the $\leq 7$ days cohort. One possible explanation is that, for the $60 \%$ of patients initiating dolutegravir-based regimens in this study, dolutegravir was coformulated with abacavir, a drug requiring testing for the HLA-B*5701 allele, which entails additional health care resources and costs and may further delay treatment initiation until results are available, although further research is warranted to confirm this hypothesis. ${ }^{41,42}$ Elvitegravir and efavirenz were also commonly used among patients included in the present study, but their relatively low genetic barrier to resistance does not make them ideal candidates for use when rapid initiation is advised. ${ }^{40}$

In the current study, PI-based regimens appeared to be more frequently used in the $\leq 7$ days cohort relative to the 8-60 days cohort, which is consistent with DHHS guidelines that recommend using pharmacologically boosted PIs, such as boosted darunavir, for patients who initiate treatment rapidly, since they have a high genetic barrier to resistance..$^{40}$ Although approved in 2018 (i.e., after the end of the study period), only the darunavir/cobicistat/tenofovir/alafenamide STR currently has phase 3 data supporting its safety and efficacy when initiated rapidly after HIV diagnosis. ${ }^{43}$

Although the results reported here largely echo those from a previous study conducted among Medicaid-insured patients, some noteworthy differences were observed. ${ }^{35}$ First, the proportion of Medicaid-insured patients initiated on an NNRTI-based regimen-which has a lower genetic barrier to resistance-was more than 10\% higher compared with commercially insured patients from the present study (i.e., $41.8 \%$ vs. $31.1 \%)$. Second, a lower proportion of Medicaid-insured patients were initiated on an STR versus commercially insured patients (i.e., $58.5 \%$ vs. $74.3 \%$ ). These observations suggest that Medicaid-insured patients are treated with medications and formulations that are not adapted to a high level of nonadherence, which was previously reported to be as high as $60 \%-80 \%$ in this population. ${ }^{32,44}$ Third, as previously reported in another study, the commercially insured population predominantly consisted of male patients, whereas the Medicaidinsured population proportionally comprised more female patients. ${ }^{32}$ In 2017, the U.S. Census Bureau reported that the median household income of same-sex male couples was 17.4\% higher than that of married opposite-sex couples and 58.8\% higher than that of unmarried opposite-sex couples. ${ }^{45}$ Thus, the higher socioeconomic status of men who have sex with men may explain the higher proportion of male patients in the commercially insured population, although this hypothesis could not be verified with the data available.

Before ART initiation, the proportion of patients with mental health-related comorbidities was higher in the 8-60 days cohort compared with the $\leq 7$ days cohort, which could reflect health care providers' reluctance to initiate ART rapidly when patients suffer from mental illnesses. This may have important implications in the treatment of HIV, because mental healthrelated comorbidities are associated with poorer adherence to ART, which in turn increases the risk of having nonsuppressed viral loads and developing drug resistance. ${ }^{46-52}$ In these patients, physicians should prioritize an ART regimen with a high genetic barrier to resistance to prevent the emergence of HIV-related symptoms and drug resistance, as recommended by current U.S. guidelines. ${ }^{40}$ In addition, efavirenz, rilpivirine, and dolutegravir may cause adverse neuropsychiatric symptoms, which might in turn contribute to lowering patients' adherence or preventing them from starting treatment rapidly, although this was not explored in the current study. ${ }^{40}$

Total accumulated health care costs were consistently lower in the $\leq 7$ days cohort versus the 8-60 days cohort at all time points analyzed. Similar trends were observed in the Medicaid study for patients initiating ART $\leq 60$ days after HIV diagnosis, although total costs were higher in commercially insured versus Medicaid-insured patients. ${ }^{31}$ This suggests that rapid ART initiation is associated with reduced costs ensuing from HIV complications. ${ }^{31}$ This is consistent with evidence from 3 randomized controlled trials, which all demonstrated that delaying ART initiation by only 2-4 weeks led to poorer viral suppression compared with same-day or rapid initiation. . $^{15,17,19}$

In support of this hypothesis, PPPM medical costs of patients in the $8-60$ days cohort were $27.1 \%$ higher than those of patients in the $\leq 7$ days cohort after 6 months of follow-up, but this difference fell to $8.1 \%$ after 36 months. This suggests that patients who deferred ART incurred high medical costs, potentially due to HIV-related complications that were left untreated, and that initiating ART subsequently reduced these costs. This is consistent with the results from the Medicaid study for patients who did not defer ART initiation for $>60$ days; medical costs were $33.0 \%$ higher in patients initiating ART 15-60 days after HIV diagnosis after 6 months versus those initiating ART $\leq 7$ days after HIV diagnosis and only 10.1\% higher after 36 months. ${ }^{31}$ Thus, the data presented in the current study show that the WHO recommendation to initiate ART $\leq 7$ days after diagnosis may lead to savings for payers. 


\section{Limitations}

The current study is subject to some limitations. Health care claims data may contain billing inaccuracies or omissions in diagnoses and other variables, but this is not expected to differ across study cohorts. Moreover, the Truven databases predominantly included commercially insured patients residing in the South. Thus, results may not be generalizable to the overall U.S. population, patients with other insurance plans, or uninsured patients. Furthermore, the time between infection and first HIV diagnosis was not available in the data. In addition, the reported proportion of PLWH initiating ART $\leq 7$ days after diagnosis was overestimated since patients with delays $>60$ days and those without evidence of treatment were excluded. Reported costs only include those incurred by the payer.

While guidelines recommend that laboratory tests be performed before initiating ART (e.g., screening for hepatitis B and hepatitis C, CD4 cell count, and urinalysis), patient selection was only based on the presence of diagnosis claims. ${ }^{53}$ It is also important to note that patients initiated on a specific ART regimen may have discontinued treatment at some point during the observation period, but cost analyses were performed based on the intent-to-treat approach. Moreover, the duration of the washout period used to identify the first observed HIV diagnosis changed from one patient to another due to the varying length of continuous eligibility in the pre-index period (although a minimum of $\geq 6$ months was required). Additionally, most patients included in the present study initiated ART before the publication of new guidelines that recommend rapid ART initiation. Finally, the present study was descriptive in nature; ART discontinuation and differences in patient characteristics across cohorts before ART initiation were not accounted for.

\section{Conclusions}

In this retrospective study of commercially insured PLWH treated with ART $\leq 60$ days after HIV diagnosis, less than $20 \%$ initiated ART $\leq 7$ days as recommended by current WHO guidelines. More patients initiating ART 8-60 days after diagnosis had mental health-related comorbidities compared with those initiating ART $\leq 7$ days. Given the known association between mental health-related comorbidities and nonadherence to ART, this suggests that patients who defer ART initiation may be at higher risk of nonadherence and may need ART regimens with a high genetic barrier to resistance as recommended by current U.S. guidelines. ${ }^{40,46-48}$ Regardless of the length of follow-up considered, total accumulated and PPPM costs were consistently lower among patients treated in the $\leq 7$ days cohort relative to those treated in the 8-60 days cohort, suggesting that rapid initiation of ART may be associated with cost savings for payers.

\section{Authors}

CARMELA BENSON, MS, MSHP; NEETA TANDON, MA; WING CHOW, PharmD, MPH; and KEITH DUNN, PharmD, BCPS, AAHIVE, Janssen Scientific Affairs, Titusville, New Jersey. BRUNO EMOND, MSc; PATRICK LEFEBVRE, MA; MARIE-HÉLÈNE LAFEUILLE, MA; and AURÉLIE CÔTÉ-SERGENT, MSc, Analysis Group, Montréal, Québec, Canada.

AUTHOR CORRESPONDENCE: Bruno Emond, MSc, Analysis Group, 1190 avenue des Canadiens-de-Montréal, Tour Deloitte, Ste. 1500, Montréal, 2C H3B 0G7, Canada. Tel.: 514.394.4455; E-mail: bruno.emond@analysisgroup.com.

\section{DISCLOSURES}

This study was supported by Janssen Scientific Affairs, which was involved in the study design, interpretation of results, manuscript preparation, and publication decisions. Emond, Lefebvre, Lafeuille, and Côté-Sergent are employees of Analysis Group, a consulting company that was contracted by Janssen Scientific Affairs to conduct this study and develop the manuscript. Benson, Tandon, Chow, and Dunn are employees of Janssen Scientific Affairs and stockholders of Johnson \& Johnson.

Part of the material in this study has been presented at the AMCP 2019 Annual Meeting; March 25-28, 2019; San Diego, CA.

\section{REFERENCES}

1. Deeks SG, Overbaugh J, Phillips A, Buchbinder S. HIV infection. Nat Rev Dis Primers. 2015;1:15035.

2. United Nations Programme on HIV/AIDS. Key populations atlas. 2018. Available at: http://www.aidsinfoonline.org/kpatlas/\#/home. Accessed October 24, 2019

3. Bavinton BR, Pinto AN, Phanuphak N, et al. Viral suppression and HIV transmission in serodiscordant male couples: an international, prospective, observational, cohort study. Lancet HIV. 2018;5(8):e438-e47.

4. Cohen MS, Chen YQ, McCauley M, et al. Prevention of HIV-1 infection with early antiretroviral therapy. N Engl J Med. 2011;365(6):493-505.

5. Farnham PG, Gopalappa C, Sansom SL, et al. Updates of lifetime costs of care and quality-of-life estimates for HIV-infected persons in the United States: late versus early diagnosis and entry into care. J Acquir Immune Defic Syndr. 2013;64(2):183-89.

6. Quinn TC, Wawer MJ, Sewankambo N, et al. Viral load and heterosexual transmission of human immunodeficiency virus type 1. Rakai Project Study Group. N Engl J Med. 2000;342(13):921-29.

7. Rodger AJ, Cambiano V, Bruun T, et al. Sexual activity without condoms and risk of HIV transmission in serodifferent couples when the HIV-positive partner is using suppressive antiretroviral therapy. JAMA. 2016;316(2):171-81.

8. Fischl MA, Richman DD, Grieco MH, et al. The efficacy of azidothymidine (AZT) in the treatment of patients with AIDS and AIDS-related complex. A double-blind, placebo-controlled trial. N Engl J Med. 1987;317(4):185-91.

9. United Nations Programme on HIV/AIDS. 90-90-90 - An ambitious treatment target to help end the AIDS epidemic. Available at: http://www.unaids.org/ sites/default/files/media_asset/90-90-90_en.pdf. Accessed October 24, 2019.

10. Egger M, May M, Chene G, et al. Prognosis of HIV-1-infected patients starting highly active antiretroviral therapy: a collaborative analysis of prospective studies. Lancet. 2002;360(9327):119-29. 
11. Strategies for Management of Antiretroviral Therapy (SMART) Study Group. Major clinical outcomes in antiretroviral therapy (ART)-naive participants and in those not receiving ART at baseline in the SMART study. J Infect Dis. 2008;197(8):1133-44.

12. Temprano ANRS 12136 Study Group. A trial of early antiretrovirals and isoniazid preventive therapy in Africa. N Engl J Med. 2015;373(9):808-22.

13. INSIGHT START Study Group. Initiation of antiretroviral therapy in early asymptomatic HIV infection. N Engl J Med. 2015;373(9):795-807.

14. Grinsztejn B, Hosseinipour MC, Ribaudo HJ, et al. Effects of early versus delayed initiation of antiretroviral treatment on clinical outcomes of HIV-1 infection: results from the phase 3 HPTN 052 randomised controlled trial. Lancet Infect Dis. 2014;14(4):281-90.

15. Amanyire G, Semitala FC, Namusobya J, et al. Effects of a multicomponent intervention to streamline initiation of antiretroviral therapy in Africa: a stepped-wedge cluster-randomised trial. Lancet HIV. 2016;3(11):e539-e48.

16. Ford N, Migone C, Calmy A, et al. Benefits and risks of rapid initiation of antiretroviral therapy. AIDS. 2018;32(1):17-23.

17. Koenig SP, Dorvil N, Devieux JG, et al. Same-day HIV testing with initiation of antiretroviral therapy versus standard care for persons living with HIV: a randomized unblinded trial. PLoS Med. 2017;14(7):e1002357.

18. Labhardt ND, Ringera I, Lejone TI, et al. Effect of offering same-day ART vs usual health facility referral during home-based HIV testing on linkage to care and viral suppression among adults with HIV in Lesotho: the CASCADE randomized clinical trial. JAMA. 2018;319(11):1103-12.

19. Rosen S, Maskew M, Fox MP, et al. Initiating antiretroviral therapy for HIV at a patient's first clinic visit: the RapIT randomized controlled trial. PLoS Med. 2016;13(5):e1002015.

20. World Health Organization. Guidelines for managing advanced HIV disease and rapid initiation of antiretroviral therapy. 2017. Available at: https:// www.who.int/hiv/pub/guidelines/advanced-HIV-disease/en/. Accessed November 14, 2019.

21. Centers for Disease Control and Prevention. Understanding the HIV care continuum. 2019. Available at: https://www.cdc.gov/hiv/pdf/library/factsheets/cdc-hiv-care-continuum.pdf. Accessed October 24, 2019.

22. Dodd PJ, Garnett GP, Hallett TB. Examining the promise of HIV elimination by 'test and treat' in hyperendemic settings. AIDS. 2010;24(5):729-35.

23. Kretzschmar ME, Schim van der Loeff MF, Birrell PJ, De Angelis D, Coutinho RA. Prospects of elimination of HIV with test-and-treat strategy. Proc Natl Acad Sci U S A. 2013;110(39):15538-43.

24. Maddali MV, Dowdy DW, Gupta A, Shah M. Economic and epidemiological impact of early antiretroviral therapy initiation in India. J Int AIDS Soc. 2015;18:20217.

25. Drabo EF, Hay JW, Vardavas R, Wagner ZR, Sood N. A cost-effectiveness analysis of preexposure prophylaxis for the prevention of HIV among Los Angeles county men who have sex with men. Clin Infect Dis. 2016;63(11):1495-1504.

26. Wagner BG, Blower S. Universal access to HIV treatment versus universal 'test and treat': transmission, drug resistance \& treatment costs. PLoS One. 2012;7(9):e41212

27. Sax PE, Sloan CE, Schackman BR, et al. Early antiretroviral therapy for patients with acute AIDS-related opportunistic infections: a cost-effectiveness analysis of ACTG A5164. HIV Clin Trials. 2010;11(5):248-59.

28. Jakubowski A, Snyman K, Kwarisiima D, et al. High CD4 counts associated with better economic outcomes for HIV-positive adults and their HIV-negative household members in the SEARCH Trial. PLoS One. 2018;13(6):e0198912.

29. Merito M, Pezzotti P; ICONA Study Group. Comparing costs and effectiveness of different starting points for highly active antiretroviral therapy in HIV-positive patients. Evidence from the ICONA cohort. Eur J Health Econ. Mar 2006;7(1):30-36
30. Siregar AY, Tromp N, Komarudin D, et al. Costs of HIV/AIDS treatment in Indonesia by time of treatment and stage of disease. BMC Health Serv Res. 2015;15:440.

31. Benson C, Emond B, Romdhani H, et al. P284: Rapid initiation of antiretroviral treatment following diagnosis of human immunodeficiency virus among Medicaid-covered patients: a real-world evaluation. Presented at: HIV Drug Therapy 2018; October 28-31, 2018; Glasgow, UK.

32. Kangethe A, Polson M, Lord TC, Evangelatos T, Oglesby A. Real-world health plan data analysis: key trends in medication adherence and overall costs in patients with HIV. J Manag Care Spec Pharm. 2019;25(1):88-93. Available at: https://www.jmcp.org/doi/10.18553/jmcp.2019.25.1.088.

33. Department of Health and Human Services. Part 36-indemnification of HHS employees. Available at: https://www.govinfo.gov/content/pkg/CFR2016-title45-voll/pdf/CFR-2016-title45-voll-part46.pdf. Accessed October 24, 2019.

34. Bureau of Labor Statistics. Consumer Price Index. Available at: https://www. bls.gov/cpi/tables/supplemental-files/home.htm. Accessed October 24, 2019.

35. Benson C, Emond B, Romdhani H, et al. B4: Long-term benefits of rapid antiretroviral therapy initiation among Medicaid-covered patients with human immunodeficiency virus. Presented at: Academy of Managed Care \& Specialty Pharmacy (AMCP) Annual Meeting; March 25-28, 2019; San Diego, CA.

36. World Health Organization. Antiretrovial therapy for HIV infection in adults and adolescents. 2010. Available at: https://apps.who.int/iris/bitstream/handle/10665/44379/9789241599764_eng.pdf;jsessionid=41E5DE55 87A3C8577CFAD9C2F7EB6CAB?sequence=1. Accessed October 24, 2019.

37. Severe P, Juste MA, Ambroise A, et al. Early versus standard antiretroviral therapy for HIV-infected adults in Haiti. N Engl J Med. 2010;363(3):257-65.

38. World Health Organization. Consolidated guidelines on the use of antiretroviral drugs for treating and preventing HIV infection. 2013. Available at: https://apps.who.int/iris/bitstream/handle/10665/85321/9789241505727_ eng.pdf?sequence=1. Accessed October 24, 2019.

39. World Health Organization. Guideline on when to start antiretroviral therapy and on pre-exposure prophylaxis for HIV. 2015. Available at: https:// apps.who.int/iris/bitstream/handle/10665/186275/9789241509565_eng. pdf?sequence=1. Accessed October 24, 2019.

40. Panel on Antiretroviral Guidelines for Adults and Adolescents; Department of Health and Human Services. Guidelines for the use of antiretroviral agents in adults and adolescents living with HIV. Available at: http://www.aidsinfo.nih.gov/ContentFiles/AdultandAdolescentGL.pdf. Accessed October 24, 2019.

41. U.S. Food and Drug Administration. Product information: TRIUMEQ (abacavir, dolutegravir, and lamivudine tablets). Available at: https://www. accessdata.fda.gov/drugsatfda_docs/label/2018/205551s014s015lbl.pdf. Accessed October 24, 2019.

42. Mallal S, Phillips E, Carosi G, et al. HLA-B*5701 screening for hypersensitivity to abacavir. N Engl J Med. 2008;358(6):568-79.

43. Huhn G, Crofoot G, Gathe Jr J, et al. Darunavir/cobicistat/emtricitabine/ tenofovir alafenamide (D/C/F/TAF) in a test-and-treat model of care for HIV-1 infection: interim analysis of the DIAMOND study. Presented at: AIDS 2018; July 23-27, 2018; Amsterdam, Netherlands.

44. Dunn K, Lafeuille M-H, Jiao X, et al. Risk factors, health care resource utilization, and costs associated with nonadherence to antiretrovirals in Medicaid-insured patients with HIV. J Manag Care Spec Pharm. 2018;24(10):1040-51. Available at: https://www.jmcp.org/doi/full/10.18553/ jmcp.2018.17507.

45. U.S. Census Bureau. Characteristics of same-sex couple households: 2005 to present. 2017. Available at: https://www.census.gov/data/tables/ time-series/demo/same-sex-couples/ssc-house-characteristics.html. Accessed November 14, 2019. 
Rapid Initiation of Antiretroviral Therapy Following Diagnosis of Human

Immunodeficiency Virus Among Patients with Commercial Insurance Coverage

46. Adejumo O, Oladeji B, Akpa O, et al. Psychiatric disorders and adherence to antiretroviral therapy among a population of HIV-infected adults in Nigeria. Int J STD AIDS. 2016;27(11):938-49.

47. Mellins CA, Havens JF, McDonnell C, et al. Adherence to antiretroviral medications and medical care in HIV-infected adults diagnosed with mental and substance abuse disorders. AIDS Care. 2009;21(2):168-77.

48. Monroe AK, Rowe TL, Moore RD, Chander G. Medication adherence in HIV-positive patients with diabetes or hypertension: a focus group study. BMC Health Serv Res. 2013;13:488.

49. Nyaku M, Beer L, Shu F. Non-persistence to antiretroviral therapy among adults receiving HIV medical care in the United States. AIDS Care. 2018:1-10.
50. Harrigan PR, Hogg RS, Dong WW, et al. Predictors of HIV drug-resistance mutations in a large antiretroviral-naive cohort initiating triple antiretroviral therapy. J Infect Dis. 2005;191(3):339-47.

51. Masikini P, Mpondo BC. HIV drug resistance mutations following poor adherence in HIV-infected patient: a case report. Clin Case Rep. 2015;3(6):353-56

52. Meresse M, March L, Kouanfack C, et al. Patterns of adherence to antiretroviral therapy and HIV drug resistance over time in the Stratall ANRS 12110/ESTHER trial in Cameroon. HIV Med. 2014;15(8):478-87.

53. U.S. Department of Health and Human Services. Guidelines for the use of antiretroviral agents in adults and adolescents living with HIV. 2018. Available at: https://aidsinfo.nih.gov/guidelines/html/l/adult-and-adolescentarv/0. Accessed October 24, 2019. 
Rapid Initiation of Antiretroviral Therapy Following Diagnosis of Human Immunodeficiency Virus Among Patients with Commercial Insurance Coverage

\section{APPENDIX Study Design Scheme}

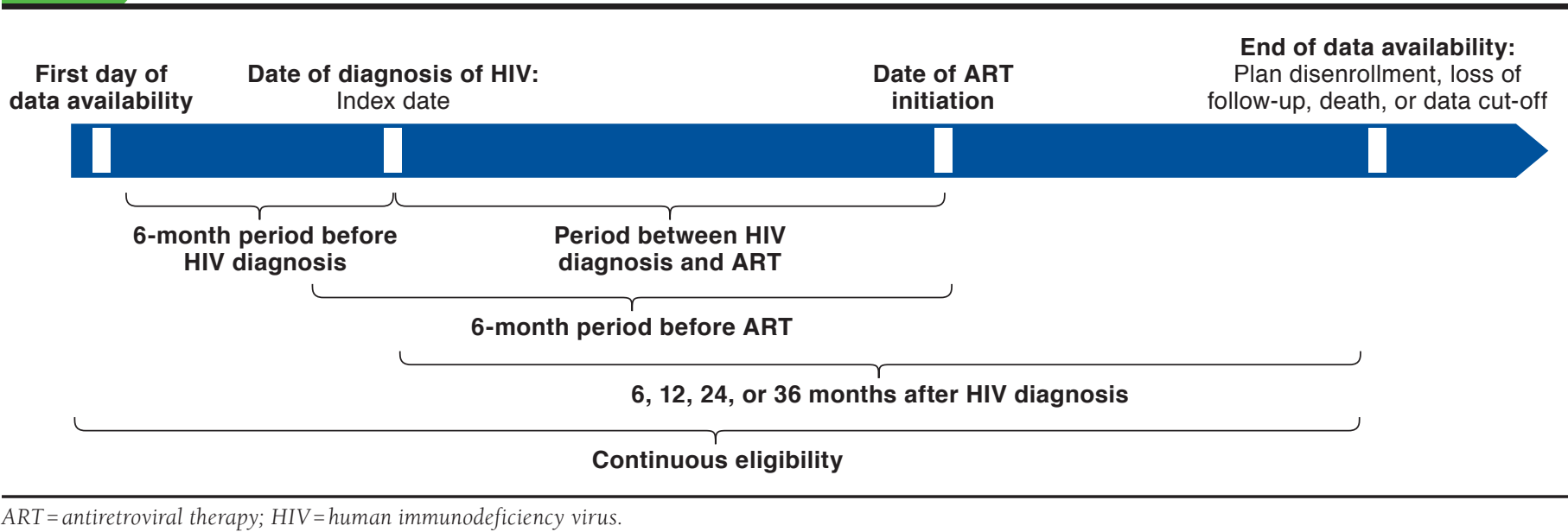

ART = antiretroviral therapy; HIV=human immunodeficiency virus. 